\title{
O conhecimento produzido acerca de climatério, família e envelhecimento
}

\author{
Knowledge produced on climacteric, family and aging \\ El conocimiento producido sobre climaterio, familia y envejecimiento
}

\author{
Glaucimara Riguete de Souza Soares'; Selma Petra Chaves Sáll; Rose Mary Costa Rosa Andrade Silvall'; \\ Ivis Emília Oliveira Souza" ; Lucia Helena Garcia Pennav'; Marcele Zveiter ${ }^{\text {vl }}$
}

\begin{abstract}
RESUMO
Objetivo: analisar o conhecimento produzido acerca de climatério, família e envelhecimento. Método: revisão integrativa da literatura, realizada na base de dados BVS, Pubmed e Portal de Periódicos Capes (2012-2017). Resultados: respeitando-se os critérios de inclusão, 22 artigos foram selecionados e analisados em duas categorias: a influência da família no processo de envelhecimento de mulheres em fase de climatério e o enfrentamento do climatério e suas desordens na natureza físico-psicológica da mulher. Conclusão: é necessário estudar o climatério para além da sintomatologia clínica da menopausa na perspectiva de se promover o envelhecimento ativo e saudável. A família é parte integrante deste processo, por isso, enfermeiros e demais profissionais da equipe multidisciplinar devem incluí-la no processo de conhecimento, entendimento, acompanhamento e cura das sintomatologias transitórias que possam acometer a mulher em fase de climatério/ menopausa.
\end{abstract}

Descritores: Climatério; família; envelhecimento; enfermagem.

\begin{abstract}
Objective: to examine the knowledge produced on climacterium, family and aging. Method: this integrative literature review used the VHL, Pubmed and Capes Journal Portal databases (2012-2017). Results: 22 articles were selected and analyzed by two categories: the family's influence on the aging process of women in the climacteric phase, and coping with the climacterium and its physical and psychological disorders. Conclusion: study of the climacterium needs to go beyond the clinical symptomatology of menopause, with a view to promoting active, healthy aging. The family is an integral part of this process and, accordingly, nurses and other professionals in multidisciplinary teams should include it in the process of learning about, understanding, following up on and curing of transitory symptoms that can affect women in the menopause / climacteric phase.

Descriptors: Climacteric; family; aging; nursing.
\end{abstract}

\section{RESUMEN}

Objetivo: analizar el conocimiento producido sobre climaterio, familia y envejecimiento. Método: revisión integrativa de la literatura, realizada en la base de datos BVS, Pubmed y Portal de Periódicos Capes (2012-2017). Resultados: respetando los criterios de inclusión, se seleccionaron 22 artículos que fueron analizados en dos categorías: la influencia de la familia en el proceso de envejecimiento de mujeres en fase de climaterio y el enfrentamiento del climaterio y sus desórdenes en la naturaleza físico-psicológica de la mujer. Conclusión: es necesario estudiar el climaterio más allá de la sintomatología clínica de la menopausia con vistas a promover el envejecimiento activo y saludable. La familia es parte integrante de este proceso, por eso, enfermeros y demás profesionales del equipo multidisciplinario deben incluirla en el proceso de conocimiento, entendimiento, acompañamiento y curación de las sintomatologías transitorias que puedan acometer a la mujer en fase de climaterio / menopausia.

Descriptores: Climaterio; familia; envejecimiento; enfermería.

\section{INTRODUÇÃO}

Em 2018, a população brasileira tem, aproximadamente, 280 milhões de pessoas, sendo 50,67\% composta por mulheres ${ }^{1}$. Nesse contexto, o envelhecimento feminino sinaliza o desafio de priorizar medidas educacionais de prevenção e promoção da saúde como meta para melhor qualidade e expectativa de vida.

A qualidade de vida se refere ao interesse pela vida e sempre esteve presente na humanidade. Por- tanto, qualidade de vida não é algo a ser alcançado, mas a ser incorporado ao cotidiano a partir do esforço e da dedicação individual e/ ou coletiva. Entende-se que o importante para a vida é cada um buscar uma boa qualidade frente às suas possibilidades de ação².

A repercussão alcançada pelo envelhecimento propiciou que a Organização Mundial da Saúde a apresentasse como prioridade política e trouxesse à luz o conceito

'Glaucimara Riguete de Souza Soares. Enfermeira; Doutora pela Escola de Enfermagem Aurora de Afonso Costa. Universidade Federal Fluminense. Brasil. E-mail: glau riguete@hotmail.com

"Selma Petra Chaves Sá; Doutora em Enfermagem. Enfermeira. Docente, Universidade Federal Fluminense. Brasil. E-mail: selmapetrasa@gmail.com "'Rose Mary Costa Rosa Andrade Silva; Enfermeira. Doutora em Enfermagem. Docente, Universidade Federal Fluminense. Brasil. E-mail: roserosauff@gmail.com IVIvis Emília Oliveira Souza;Enfermeira; Doutora em Enfermagem Docente, Universidade Federal do Rio de Janeiro. Brasil. E-mail: ivis@superig.com.br `Lucia Helena Garcia Penna. Enfermeira; Doutora em Enfermagem. Docente de Enfermagem, Universidade do Estado do Rio de Janeiro. Brasil. E-mail: luciapenna@terra.com.br v'Marcele Zveiter; Doutora em Enfermagem. Enfermeira. Docente de Enfermagem, Universidade do Estado do Rio de Janeiro. Brasil. E-mail: marcelezveiter@hotmail.com 
de envelhecimento ativo, que se aplica a indivíduos e grupos populacionais, caracterizando-se como um processo de otimização das oportunidades de saúde, participação e segurança, e tendo por objetivo melhorar a qualidade de vida à medida que as pessoas envelhecem. Hoje há uma feminização do envelhecimento, pois mulheres vivem pelo menos 4,5 anos mais que homens ${ }^{3}$.

Considerando a mudança no perfil dos usuários em saúde, no que diz respeito a envelhecimento e longevidade, destaca-se que profissionais, notadamente enfermeiros, devam estar atentos a essa valorização para além do funcionamento biológico, propondo medidas de prevenção e promoção da saúde que se integrem às diversas linhas de cuidado em saúde da mulher.

A atenção ao climatério e à menopausa é uma linha de cuidado que precisa ser percebida e entendida, não só pela mulher e por quem convive ao seu redor, mas também por profissionais da saúde, como parte longínqua do envelhecimento. A mulher procura os serviços de saúde para cuidar de outros, porém precisa também cuidar de si. Considerar as implicações da identidade feminina ao longo do ciclo vital é desafiador, pois tanto o climatério quanto o envelhecimento liberam novos sentimentos, envolvendo perdas e ganhos ${ }^{4}$. Neste estudo, pesquisar-se-á o climatério para além do contexto do ser mulher que vivencia o fenômeno.

O climatério é uma fase biológica da vida, marca a transição entre o período reprodutivo e o não reprodutivo, sendo a menopausa seu marco fisiológico, em torno dos 48 aos 50 anos de idade. Quando essa fase se apresenta sintomática, chama-se síndrome do climatério, podendo acarretar mudanças de ordem emocional, social e física na vida da mulher ${ }^{5}$.

O cuidado da enfermagem é essencial nesse momento da vida da mulher, pois é quando os filhos saem de casa e a mulher reconhece a impossibilidade fisiológica da maternidade por conta da ausência menstrual $^{6}$. Tais fatos podem gerar conflitos entre mulher e famílias - termo empregado no plural, pois remete ao fato de que está em declínio a ideia de família tradicional, cultivante da mulher dona de casa.

Vale lembrar que o contexto familiar sofreu transformações significativas no que diz respeito à presença da mulher: deixou-se de conviver com a mãe e executora das tarefas do lar e passou-se a conviver com uma mulher que além de procriar e cuidar da casa também trabalha fora, ascende socialmente, vive uma sexualidade libertadora e, por vezes, é a chefe da família. Nesse sentido, percebe-se que, para significar a fase do climatério/ menopausa de uma mulher mergulhada na contemporaneidade, não se deve pensar em família-indivíduos que compõem um mesmo núcleo, numa a mesma casa -, e sim em famílias - aliança entre grupos que dão ao ser humano a possibilidade de se fazer social ${ }^{7}$.

A questão norteadora do estudo foi: que conhecimentos científicos têm sido produzidos acerca da interseção entre os conceitos de climatério, família e envelhecimento? Assim, o objetivo desta revisão de literatura foi analisar o conhecimento produzido acerca de climatério, família e envelhecimento.

\section{Metodologia}

A metodologia adotada para este estudo é a revisão integrativa de literatura, que objetiva sintetizar resultados sobre um tema ou questionamento de forma sistematizada e abrangente para fornecer subsídios amplos a determinado assunto ou problema abordado ${ }^{8}$. Desse modo, nesta revisão, buscou-se artigos em formato eletrônico, relacionados ao tema climatério, família e envelhecimento, com o objetivo de analisar que conhecimentos têm sido produzidos acerca dessa temática.

Para tanto, foram seguidas as seguintes etapas: identificação do tema, busca na literatura divulgada em meio eletrônico, categorização e avaliação dos estudos compreendidos na revisão integrativa, interpretação dos resultados e, por fim, síntese do conhecimento evidenciado nas pesquisas analisadas.

A seleção obedeceu aos seguintes critérios de inclusão: produções em formato de artigos, cujos descritores fossem climatério, família e envelhecimento (booleano $A N D$, via Decs/ Mesh-Terms), em formato texto completo, com publicação nos últimos 5 anos e títulos com aderência à temática. Como critério de exclusão, consideraram-se artigos sem aderência à questão de pesquisa.

A seguir, são apresentadas as fontes selecionadas e os respectivos quantitativos: Biblioteca Virtual em Saúde (um artigo - BDENF), PUBMED (cinco artigos) e Portal de Periódicos Capes: CINAHL (seis artigos), SCOPUS (seis artigos), EBSCO (seis artigos) e WEB OF SICENSE (dois artigos).

Após leitura aprofundada, procedeu-se à análise de conteúdo, proposta por Bardin ${ }^{9}$, das ideias principais sobre as temáticas presentes nos artigos. Os dados foram operacionalizados seguindo as etapas de pré-análise, leitura flutuante e em profundidade, codificação, tratamento dos resultados e interpretação. Foram consideradas duas categorias: a influência da família no processo de envelhecimento de mulheres em fase de climatério e o enfrentamento do climatério e suas desordens na natureza físico-psicológica da mulher.

\section{RESUltados E Discussão}

O fenômeno do envelhecimento ganhou representatividade social no cenário brasileiro, implicando em maior concentração de publicações na área da saúde, principalmente enfermagem e psicologia ${ }^{10}$.

Das 22 produções selecionadas ${ }^{11-32}, 15$ foram realizadas no Brasil e sete no exterior. Importante ressaltar a prevalência de estudos brasileiros disponibilizados em bases de dados internacionais. Apreende-se equivalência na natureza dos estudos, sendo 10 de abordagem quantitativa, 10 de abordagem qualitativa, à execução de e dois de abordagem mista, conforme mostra a Figura 1. 


\begin{tabular}{|c|c|c|}
\hline Título do estudo & Origem dos estudos & $\begin{array}{l}\text { Abordagem } \\
\text { metodológica }\end{array}$ \\
\hline $\begin{array}{l}\text { Grand mothering and cognitive resources are required for the } \\
\text { emergence of menopause and extensive post-reproductive } \\
\text { lifespan }{ }^{11} \text {. }\end{array}$ & Montepellier, França & Quantitativa \\
\hline $\begin{array}{l}\text { Men's perceptions and attitudes toward their wives experiencing } \\
\text { menopause. J Women Aging }{ }^{12} \text {. }\end{array}$ & SP, Brasil & Qualitativa \\
\hline $\begin{array}{l}\text { Potenciais interações relacionadas ao uso de medicamentos, } \\
\text { plantas medicinais e fitoterápicos em mulheres no período do } \\
\text { climatério }{ }^{13} \text {. }\end{array}$ & RS, Brasil & Quantitativa \\
\hline $\begin{array}{l}\text { Prevalence and factors associated with urinary incontinence in } \\
\text { climacteric }^{14} \text {. }\end{array}$ & SP, Brasil & Quantitativa \\
\hline $\begin{array}{l}\text { Osteoporose na atenção primária: uma oportunidade para } \\
\text { abordar os fatores de risco }{ }^{15} \text {. }\end{array}$ & Maranhão, Brasil & Quantitativa \\
\hline Autoavaliação vocal de mulheres na menopausa ${ }^{16}$. & Paraná, Brasil & Quantitativa \\
\hline $\begin{array}{l}\text { Estudio sobre la resiliencia en ancianas del Valle de Paraíba, São } \\
\text { Paulo, Brasil|17. }\end{array}$ & SP, Brasil & Quantitativa \\
\hline $\begin{array}{l}\text { Sex life and role identity in Taiwanese women during } \\
\text { menopause: a qualitative study }{ }^{18} \text {. }\end{array}$ & Taiwan, China & Qualitativa \\
\hline $\begin{array}{l}\text { Menopausal symptons and quality of life among Saudi women } \\
\text { visiting primary care clinics in Ryadhi, Saudi Arabia }{ }^{19} \text {. }\end{array}$ & Riyadh, Arábia Saudita & Quantitativa \\
\hline $\begin{array}{l}\text { O impacto da menopausa nas relações e nos papéis sociais } \\
\text { estabelecidos na família e no trabalho }{ }^{20} \text {. }\end{array}$ & $\begin{array}{l}\text { Uberlândia, MG } \\
\text { Brasil }\end{array}$ & Mista \\
\hline $\begin{array}{l}\text { Influências do climatério para o envelhecimento na percepção de } \\
\text { mulheres idosas: subsídios para a enfermagem }{ }^{21} \text {. }\end{array}$ & RJ, Brasil & Qualitativa \\
\hline $\begin{array}{l}\text { Contextualização do envelhecimento saudável na produção } \\
\text { científica brasileira } 22 \text {. }\end{array}$ & Paraíba, Brasil & Mista \\
\hline $\begin{array}{l}\text { Role of grand parenting in postmenopausal women's cognitive } \\
\text { health: results from the Women's Healthy Aging Project }{ }^{23} \text {. }\end{array}$ & Victoria, Australia & Quantitativa \\
\hline $\begin{array}{l}\text { Climateric manifestations most frequent among women of a } \\
\text { family health unit }{ }^{24} \text {. }\end{array}$ & Pernambuco, Brasil & Quantitativa \\
\hline Influências das famílias no cuidado às mulheres climatéricas ${ }^{25}$. & Ceará, Brasil & Qualitativa \\
\hline $\begin{array}{l}\text { O homem também fala: o climatério feminino na ótica } \\
\text { masculina }{ }^{26} \text {. }\end{array}$ & RS, Brasil & Qualitativa \\
\hline $\begin{array}{l}\text { Representações sociais da vida sexual de mulheres no climatério } \\
\text { atendidas em serviços públicos de saúde }{ }^{27} \text {. }\end{array}$ & RJ, Brasil & Qualitativa \\
\hline $\begin{array}{l}\text { Ações de atenção primária dirigidas às mulheres de } 45 \text { a } 60 \text { anos } \\
\text { de idade }{ }^{28} \text {. }\end{array}$ & SP, Brasil & Qualitativa \\
\hline $\begin{array}{l}\text { Fisioterapia gerontológica na atenção primária à saúde: uma } \\
\text { experiência na região norte }{ }^{29} \text {. }\end{array}$ & Pará, Brasil & Qualitativa \\
\hline $\begin{array}{l}\text { The menopause transition experiences of Chinese Singaporean } \\
\text { women: an exploratory qualitative study }{ }^{30} \text {. }\end{array}$ & Singapura, Ásia & Qualitativa \\
\hline Increased longevity evolves from grandmothering ${ }^{31}$. & Sidnei, Austrália & Quantitativa \\
\hline $\begin{array}{l}\text { Toward healthy aging through empowering self-care during } \\
\text { the climacteric stage }{ }^{32} \text {. }\end{array}$ & $\begin{array}{l}\text { Cidade do México, } \\
\text { México }\end{array}$ & Qualitativa \\
\hline
\end{tabular}

FIGURA 1: Artigos selecionados segundo título, origem dos estudos e abordagem metodológica. Período 2012-2017.

As categorias emergentes desta revisão integrativa são tratadas a seguir.

\section{A influência da família no processo de envelhecimento de mulheres em fase de climatério}

A análise da influência da família enquanto participante do envelhecimento feminino no climatério destacou a correlação entre a menopausa, o cessar definitivo da ovulação e o fim da expectativa de vida. Foram discutidas a Hipótese Materna, que versa sobre a regulação da fertilidade a partir da menopausa em sua evolução natural, defendendo que essa regulação deva acontecer, já que quanto mais velha fica uma mulher, maior é seu risco de morrer no parto ${ }^{11}$; e a Hipótese da Avó, que traz uma abertura à longevidade quando subsidiada a fertilidade de suas filhas através da previsão dos netos, sendo esta uma possibilidade de se viver fértil por muitos anos ${ }^{12}$. Para além da reprodução, as avós são presenças capazes de promover a cognição e o engajamento social de suas filhas, quando oferecem assistência e companhia a seus netos ${ }^{13}$.
A expectativa de vida elevada é a promissora desta oportunidade de interação entre mães, filhas e netos. Uma vida mais longa supera os efeitos deletérios do envelhecimento a partir da adoção de cuidados necessários frente às alterações ocasionadas pelo tempo ${ }^{33}$. Ter uma passagem agradável por toda a fase do climatério é condição fundamental para o envelhecimento saudável. Neste cenário, reforça-se a importância de fomentar políticas que abracem os princípios de se envelhecer ativamente. É essencial ao indivíduo que envelhece acumular, desde cedo, saúde e capitais intelectual, social e financeiro. É imperativo que o setor público, a academia, as instituições da sociedade civil, o setor privado e a mídia ofertem essa assistência ${ }^{33}$.

Comumente, mulheres idosas apontam que o climatério influencia no envelhecimento, porque inicia a senescência, e diversas são as mudanças físicas, as influências culturais, as mudanças no estilo de vida e na história pessoal e familiar que enfrentam ${ }^{21}$.

É oportuno considerar que o processo saúde-doença é um fenômeno complexo, modulado por 
condicionantes biológicos, psicológicos, culturais, econômicos e políticos; e as necessidades de saúde de quem envelhece se referem às múltiplas dimensões do real e à singularidade dos fenômenos de saúde ou doença que afetam os indivíduos e suas famílias ${ }^{34}$. A família é considerada uma instituição de amparo social, que gera possibilidades de enfrentamento para um viver com mais qualidade de vida ${ }^{25}$.

O contexto familiar pode influenciar na forma como as mulheres vivenciam o climatério. Dentre as influências positivas, destacam-se o carinho, a afetividade e a relação de identidade como primordiais para o enfrentamento do estresse. As experiências compartilhadas pelas mulheres mais velhas da família amenizam a ansiedade trazida pelas expectativas acerca do novo ${ }^{18}$. Entre as influências negativas, a falta de escuta por parte dos cônjuges pode causar conflitos. Lançar à mão estratégias que envolvam os homens no processo de cuidado das mulheres climatéricas é imprescindível para amenizar mal-entendidos ${ }^{12}$.

Os homens possuem dificuldade em verbalizar questões relativas ao climatério. Apresentam frequentemente, em suas falas, respostas pontuais; porque talvez possam se sentir incomodados por reconhecer que as companheiras precisam de apoio, mas não sabem qual conduta exercer para ajudá-las ${ }^{26}$. Aqueles que melhor compreendem suas esposas sobre as mudanças ocorridas na menopausa dão o suporte emocional necessário para melhorar a qualidade de suas relações conjugais ${ }^{12}$. Por isso, o contexto das famílias tem atraído a atenção de estudiosos e pesquisadores que visam analisar e compreender sua história, suas transformações, suas adaptações, seus modos de ser e as repercussões para um viver mais positivo ${ }^{35}$. É importante buscar e obter o apoio de familiares e amigos no climatério/ menopausa, sendo esta uma forma de experimentar melhor esse período ${ }^{30}$.

Sintomas decorrentes da menopausa e do climatério são capazes de alterar a qualidade das relações estabelecidas no trabalho e na família, influenciar o estado de satisfação das mulheres e afetar sua rotina habitual e/ou relações interpessoais. Viver o climatério sem preparo pode gerar dificuldade para o enfrentamento dos desafios que surgem e, ainda, comprometer a autoestima, os relacionamentos e a qualidade de vida ${ }^{20}$. A escassas informações acerca desse fenômeno, no Brasil, afetam mais as famílias de baixa renda. Além disso, a baixa ou nenhuma escolaridade e a ausência de procura por serviços de saúde contribuem para incompreensão da menopausa ${ }^{36}$.

No contexto da representação social, a vida sexual de mulheres no climatério se revela através dos estereótipos, representados por uma formação cultural conservadora, em que o sexo e o amor caminham de modo indissociável. Porém, o envelhecimento sexual da mulher acontece numa cultura repleta de preconceitos, medos e silêncios. Subjugada pela sociedade, ela não consegue se preparar para ter ou vivenciar uma vida sexual satisfatória e prazerosa ${ }^{27}$. Assistir à mulher no climatério, incluindo a sexualidade, deve ser uma das prioridades das Políticas Públicas de Saúde, pois muitas se preocupam com as mudanças que ocorrem em seu corpo, com o prazer sexual e com sua finalidade reprodutiva ${ }^{37}$.

O envelhecimento de mulheres em situação de climatério/menopausa se associa a um esgotamento das reservas emocionais, principalmente pela possibilidade de perdas simultâneas. Efeitos negativos, como a morte de cônjuges e amigos, causam prejuízos às mulheres no climatério quanto ao engajamento físico e psicológico ${ }^{17}$. O crescimento dos filhos e sua saída de casa podem representar grande perda, principalmente para aquelas que dedicaram sua vida inteiramente à família ${ }^{38}$. Portanto, o empoderamento feminino pode contribuir para melhorar a percepção sobre o climatério/ menopausa, bem como para a importância do cuidado de si $^{32}$.

Trabalhar o enfoque do envelhecimento saudável prevêa visualização e a defesa da pessoa que envelhece na família e na sociedade, de modo participativo e construtivo para a inclusão social em todos os aspectos. Profissionais da saúde têm a missão de se dedicar à proposta da atenção básica, que deve ser resolutiva, integral e humanizada ${ }^{22}$ Torna-se importante a inclusão de serviço psicológico na rede de saúde, que possibilite atendimento eficiente desde os sintomas iniciais do climatério/menopausa a fim de promover o envelhecimento com qualidade de vida ${ }^{38}$.

\section{Enfrentamento do climatério e suas desordens na natureza físico-psicológica da mulher}

As manifestações mais frequentes, reveladas por mulheres no período do climatério, foram melancolia e cefaleia, sendo superiores às queixas físicas como artralgia e mialgia. Quando compreendem os motivos de tais manifestações, menos intensos são os sintomas e menor é o impacto em suas vidas. Sendo bem orientadas sobre a menopausa, podem vivê-la de maneira menos sintomática, principalmente em relação aos sintomas psicológicos ${ }^{24}$. A percepção relatada pelas mulheres acerca das mudanças corporais e emocionais no climatério precisa ser escutada e compreendida, para que possam ser orientadas pelos enfermeiros, principalmente os de estratégia saúde da família, que desenvolvem consultas com ênfase na escuta e nas ações educativas ${ }^{39}$. Entretanto, nas unidades de saúde, há ausência de diretrizes específicas para as mulheres de 45 a 60 anos, sendo o atendimento feito de modo espontâneo, sem fluxo de agendamento ${ }^{28}$.

Apesar dos princípios da integralidade propostos à saúde da mulher, ainda se registra poucas atividades de promoção da saúde no climatério ${ }^{25}$. Nas ações de atenção primária, os profissionais relataram que as demandas femininas se iniciam pelos sintomas físicos da menopausa, enquanto a organização do cotidiano das unidades ainda se relaciona muito a aspectos curativos e/ ou preventivos, estando limitados ao processo saúde-doença ${ }^{28}$. 
A alteração na voz também é um sintoma físico bastante presente no climatério/ menopausa, capaz de influenciar negativamente a qualidade de vida. Quanto mais a mulher perde a potência da sua voz, maior é a exteriorização do seu estresse, pois prejudica suas relações sociais ${ }^{16}$. Há mais de uma década, no contexto da saúde pública, as campanhas de prevenção e os programas educativos voltados para o distúrbio da voz recebiam pouca aderência, mesmo dos grupos profissionais que utilizam a voz como principal instrumento de trabalho ${ }^{40}$, e isto é o reflexo da ausência de conhecimento que permanece até hoje.

Outra queixa física de elevada prevalência é a incontinência urinária, sendo importante, neste caso propor estratégias de cuidados primários à saúde. A intervenção precoce do profissional desde o pré-natal é capaz de preservar a musculatura pélvica, o que mais tardiamente vem a ser um ganho para a mulher quanto à prevenção da incontinência urinária. Este distúrbio se instala principalmente no período pós-menopausa, sendo explicado pela queda do hormônio estrogênio, que desempenha importante papel no mecanismo da continência urinária. Destaca-se que essa afecção pode se tornar incapacitante e gerar grande impacto social, econômico, físico e psicológico ${ }^{14}$.

A inserção do exercício da fisioterapia gerontológica em unidades básicas de saúde da família foi um ganho para a população que envelhece e que carrega consigo a necessidade de adaptações relacionadas, por exemplo, ao período da pós-menopausa. Durante o envelhecimento, mulheres estão susceptíveis a obesidade, diabetes, hipertensão, enfraquecimento muscular, atrofia genital e incontinência urinária como principais problemas de saúde. Desse modo, a fisioterapia em mulheres com foco na atenção primária, vem sendo uma prática favorável para a melhora física, para a autoestima e, consequentemente, vem influenciando positivamente para um melhor relacionamento social e familiar ${ }^{29}$.

A osteoporose é outra condição física capaz de desestabilizar significativamente a qualidade de vida de mulheres em situação de climatério. Caracteriza-se por uma baixa massa óssea que deixa a mulher susceptível a fraturas. Profissionais da saúde precisam estar aptos a identificar os fatores de risco na abordagem primária, pois é uma doença multifatorial com prevalência crescente na população feminina ${ }^{15}$.

As aparições físico-psicológicas do climatério/ menopausa despertam nas mulheres a busca pela cura clínica; foi realizado um estudo sobre as potenciais interações relacionadas ao uso concomitante de medicamentos, plantas medicinais e fitoterápicos em mulheres no período do climatério e constatou-se que é necessário propor estratégias de intervenção para evitar possíveis interações medicamentosas, assim como a automedicação à base de plantas medicinais e fitoterápicos ${ }^{13}$. Nesse sentido, ações que visem à sensibilização e capacitação dos profissionais responsáveis pela prescrição de medica- mentos, com relação ao uso seguro de plantas medicinais e de fitoterápicos associados ou não a medicamentos, são importantes ferramentas para o cuidado destinado a estas mulheres. Também é relevante capacitar os agentes comunitários de saúde, utilizando como instrumento de trabalho os materiais do Ministério da Saúde e as listas oficiais de plantas medicinais, com o objetivo de subsidiá-los a orientar e sanar dúvidas dos usuários sobre o assunto, bem como identificar demandas e apresentá-las às equipes de saúde ${ }^{13}$.

É do senso comum que as mulheres busquem pela associação de diversos medicamentos para tratar os sintomas decorrentes do climatério e da menopausa, acerca das mudanças percebidas na sexualidade. Parte delas relata que as mudanças físicas tornam difíceis as interações sexuais e, por isso, evitam ter vida sexual ativa, utilizando o desconforto como principal pressuposto. Outras procuram melhorar seus encontros sexuais através das modificações comportamentais ou com medicamentos que compõem a terapia de reposição hormonal. Os hormônios são sintetizados em laboratório e têm efeitos semelhantes aos hormônios produzidos pelo organismo feminino, sendo amplamente utilizados em todo o mundo. Seus potenciais efeitos adversos são tema de interesse para a saúde pública devido ao potencial impacto que possa acarretar à população exposta ${ }^{39}$. Mulheres sauditas, por exemplo, não são adeptas ao uso de hormônios sintéticos e encaram o climatério e menopausa como um avançar necessário à vida. Queixam-se menos de ondas de calor que as mulheres ocidentais, tendo estas, em destaque, as dores articulares, a exaustão física e mental ${ }^{19}$.

\section{CONCLUSÃO}

Ao se analisar a produção científica sobre climatério, família e envelhecimento, percebeu-se que há preocupação e necessidade em estudar para além da sintomatologia clínica da menopausa. As pesquisas tendem a apresentar inovações referentes à promoção da saúde, prevenção de doenças e manutenção da qualidade de vida como os pilares para o envelhecimento ativo e saudável de mulheres em fase de climatério.

Entre os determinantes reveladores, está a valorização em se entender a influência da família sobre o processo de envelhecimento e de adaptação à nova fase de vida. Nesse sentido, enfermeiros e demais profissionais da equipe multidisciplinar devem incluir os membros da família no processo de conhecimento, entendimento, acompanhamento e cura das sintomatologias transitórias que possam acometer a mulher em fase de climatério/ menopausa. A família constitui um universo de relações diferenciadas entre seus membros e precisa ser valorizada na busca por um cuidado integral.

\section{REFERÊNCIAS}

1. Instituto Brasileiro de Geografia e Estatística [site de internet]. Projeção da população do Brasil e das unidades de federação. 
[citado em 01 jan 2018]. Disponível em: http://www.ibge.gov.br/ apps/populacao/projecao/.

2. Almeida MAB, Gutierrez GL, Marques R, organizadores. Qualidade de vida: definição, conceitos e interfaces com outras áreas de pesquisa. São Paulo : EACH; 2012.

3. Centro Internacional de Longevidade Brasil. Envelhecimento ativo: um marco político em resposta à revolução da longevidade. [site de internet] [citado em 01 jan 2018]. Disponível em: http:// ilcbrazil.org/portugues/wp-content/uploads/sites/4/2015/12/Envelhecimento-Ativo-Um-Marco-Pol\%C3\%ADtico-ILC-Brasil_web.pdf 4. Silva JPL, Marcolino EC, Fernandes NMS, Moura IM, Araújo MZ. Representações do climatério e suas repercussões na vida da mulher: uma revisão sistemática. In: Anais do IV Congresso Internacional de Envelhecimento Humano; 2015; Paraíba, Brasil. Paraíba(PB):IV Congresso Internacional de Envelhecimento Humano; 2015.

5. Pereira ABS, Martins CA, Pereira MS, Lima JR, Souza ACS, Ream PSF. Atenção ao climatério realizada por profissionais da estratégia saúde da família. Rev. enferm. UERJ. 2016; 24(1):e13122.

6. Oliveira ZM, Vargens OMC, Acioli S, Santos RS. Nursing care in the climateric: demedicalizing perspective in primary health care cuidado. Rev. enferm. UFPE on line. 2017; 11(Suppl. 2):1032-43. 7. Strey MN, Silva Neto JAS, Horta RL. Família e gênero. Porto Alegre (RS): EDIPUCRS; 2007.

8. Ercole FF, Melo LS, Alcoforado CLGC. Integrative review versus systematic review. REME rev. min. enferm. 2014; 18:12-4.

9. Bardin L. Análise de conteúdo. São Paulo : Editora 70; 2011.

10. Silva MC, Ogata MN, Oliveira DC. O estado de arte das produções científicas nacionais das representações sociais do envelhecimento na perspectiva da saúde. Rev. Kairós. 2015; 18(esp. 19):49-63.

11. Aimé C, André JB, Raymond M. Grandmothering and cognitive resources are required for the emergence of menopause and extensive post-reproductive lifespan. PLoS Comput. Biol. 2017; 13(7):e1005631.

12. Rodolpho JRC, Quirino BC, Hoga LAK, Rosa PLFS. Men's perceptions and attitudes toward their wives experiencing menopause. J. Women Aging. 2016; 28(4):322-33.

13. Gelatti GT, Oliveira KR, Colet CF. Potenciais interações relacionadas ao uso de medicamentos, plantas medicinais e fitoterápicos em mulheres no período do climatério. Rev. pesqui. cuid. fundam. (Online). 2016; 8(2):4328-46.

14. Dellú MC, Schmitt ACB, Cardoso MRA, Pereira WMP, Pereira ECA, Vasconcelos ESF et al. Prevalence and factors associated with urinary incontinence in climacteric. AMB rev. Assoc. Med. Bras. 2016; 62(5):441-6.

15. Costa ALD, Silva MACN, Brito LMO, Nascimento ACB, Barbosa $\mathrm{MCL}$, Batista JE et al. Osteoporosis in primary care: an opportunity to approach risk factors. Rev. bras. reumatol. 2016; 56(2):111-6. 16. Basílio BN, Ribeiro VV, Pereira EC, Leite APD. Vocal self-assessment of women in menopause. Rev. CEFAC. 2016; 18(3):649-55. 17. Araújo CLO, Faro ACM. Estudio sobre la resiliencia en ancianas del Valle de Paraíba, São Paulo, Brasil. Enfermería Global. 2016; 15(2):63-80.

18. Yang CF, Kenney NJ, Chang TC, Chang SR. Sex life and role identity in Taiwanese women during menopause: a qualitative study. J. Adv. Nurs. 2016; 72(4):770-81.

19. AlDughaither A, AlMutairy H, AlAteeq M. Menopausal symptoms and quality of life among Saudi women visiting primary care clinics in Riyadh, Saudi Arabia. Int. J. Womens Health. 2015; 29(7):645-53.

20. Lomônaco C, Tomaz RAF, Ramos MTO. O impacto da menopausa nas relações e nos papéis sociais estabelecidos na família e no trabalho. Reprod. clim.2015; 30(2):58-66.
21. Silva GF, Moura MAV, Almeida MVS, Sá SPC, Queiroz ABA. Climacteric influence for aging in the perception of elderly women: subsidies for nursing. Rev. eletrônica enferm. 2015; 17(3):1-8.

22. Medeiros FAL, Nóbrega MML, Medeiros ACT, Bittencourt GKGD, Leite GA. Contextualization of healthy ageing in the brazilian scientific production. Rev. enferm. UFPE on line. 2015; 9(2):985-93.

23. Burn KF, Henderson VW, Ames D, Dennerstein L, Szoeke C. Role of grandparenting in postmenopausal women's cognitive health: results from the Women's Healthy Aging Project. Menopause. 2014; 21(10):1069-74.

24. Paiva ER, Silva MM, Oliveira CDB, Leal IHS, Araújo VS, Dias $M D$. Climateric manifestations most frequent among women of a family health unit. Rev. enferm. UFPE on line. 2013; 7(11):6430-7. 25. Santos JS, Fialho AVM, Rodrigues DP. Influências das famílias no cuidado às mulheres climatéricas. Rev. eletrônica enferm. 2013; 15(1):215-22.

26. Leite MT, Taschetto A, Hildebrandt LM, Van der Sand ICP. O homem também fala: o climatério feminino na ótica masculina. Rev. eletrônica enferm. 2013; 15(2):344-51.

27. Araújo IA, Queiroz ABA, Moura MAV, Penna LHG. Social representations of the sexual life of climacteric women assisted at public health services. Texto \& contexto enferm. (Online). 2013; 22(1):114-22.

28. Garcia NK, Gonçalves R, Brigagão JIM. Ações de atenção primária dirigidas às mulheres de 45 a 60 anos de idade. Rev. eletrônica enferm. 2013;15(3):713-21.

29. Nascimento RG, Oliveira JSS, Ferreira LS, Santos ZNL, Cardoso RO. Fisioterapia gerontológica na atenção primária à saúde: uma experiência na região norte. Ciência \& saúde. 2013; 6(3):222-8. 30.Lim HK, Mackey S. The menopause transition experiences of Chinese Singaporean women: an exploratory qualitative study. J. Nurs .Res. 2012; 20(2):81-90.

31. Kim PS, Coxworth JE, Hawkes K. Increased longevity evolves from grandmothering. Proc Biol Sci. 2012; 279: 4880-4.

32. Doubova SV, Infante-Castañeda C, Martinez-Vega I, PérezCuevas R. Toward healthy aging through empowering self-care during the climacteric stage. Climacteric. 2012; 15(6):563-72.

33. Kalache A. Vested rights, active aging and resiliance: the importance of such concepts throughout life. Rev. bras. geriatr. gerontol. 2017; 20(2):159-60.

34. Ciosak SI, Braz E, Costa MFBNA, Nakano NGR, Rodrigues J, Alencar RA et al. Senescence and senility: a new paradigm in Primary Health Care. Rev. Esc. Enferm. USP. 2011; 45(2):1763-68. 35. Aquino DI, Conceição CRFRT, Pereira CRC. A família contemporânea e a estratégia de saúde da família: uma revisão integrativa. Estácio Saúde. 2017; 6(1):48-62.

36. Ferreira ICC, Silva SS, Almeida RS. Menopausa, sinais e sintomas e seus aspectos psicológicos em mulheres sem uso de reposição hormonal. Ensaios Cienc., Cienc. Biol. Agrar. Saúde. 2015; 19(2):60-4.

37. Alves ERP, Costa AM, Bezerra SMMS, Nakano AMS, Cavalcanti AMTS, Dias MD. Climacteric: intensity of symptoms and sexual performance. Texto \& contexto enferm. (Online) 2015; 24(1):64-71. 38. Freitas ER, Barbosa AJG. Qualidade de vida e bem-estar psicológico no climatério. Arq. bras. psicol. 2015; 67(3):112-24. 39. Santos AAS, Silva FV, Martins FL. Percepção das mulheres no município de Paulo Afonso, na Bahia, sobre as mudanças corporais e emocionais no período do climatério. Estação Científica (UNIFAP). 2016; 6(1):91-104.

40. Machado MAMP, Aldrighi JM, Ferreira LP. The meanings attributed to the voice by menopause women. Rev. saúde pública. (Online). 2005; 39(2):261-9. 